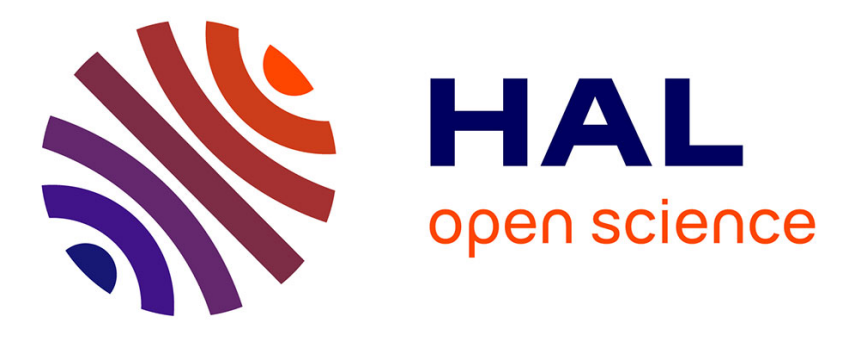

\title{
Ondes adhérence-glissement-décollement sous contact unilatéral frottant
}

\author{
Abdelbacet Oueslati, Quoc Son Nguyen, Laurent Baillet
}

\section{To cite this version:}

Abdelbacet Oueslati, Quoc Son Nguyen, Laurent Baillet. Ondes adhérence-glissement-décollement sous contact unilatéral frottant. Comptes Rendus Mécanique, 2003, 331 (2), pp.133-140. 10.1016/S1631-0721(02)00009-8 . hal-00111374

\section{HAL Id: hal-00111374 \\ https://hal.science/hal-00111374}

Submitted on 2 Apr 2018

HAL is a multi-disciplinary open access archive for the deposit and dissemination of scientific research documents, whether they are published or not. The documents may come from teaching and research institutions in France or abroad, or from public or private research centers.
L'archive ouverte pluridisciplinaire HAL, est destinée au dépôt et à la diffusion de documents scientifiques de niveau recherche, publiés ou non, émanant des établissements d'enseignement et de recherche français ou étrangers, des laboratoires publics ou privés. 


\title{
Ondes adhérence-glissement-décollement sous contact unilatéral frottant
}

\section{Stick-slip-separation waves in unilateral and frictional contact}

\author{
Abdelbacet Oueslati ${ }^{\mathrm{a}}$, Quoc-Son Nguyen ${ }^{\mathrm{a}}$, Laurent Baillet ${ }^{\mathrm{b}}$ \\ a Laboratoire de mécanique des solides, CNRS-UMR 7649, École Polytechnique, 91128 Palaiseau, France \\ ${ }^{\mathrm{b}}$ Laboratoire de mécanique des contacts, CNRS-UMR 5514, INSA-Lyon, 69621 Villeurbanne, France
}

\begin{abstract}
Résumé
Il s'agit d'une étude numérique sur la réponse dynamique d'un système de deux cylindres coaxiaux, en rotation relative avec contact unilatéral frottant suivant la loi de Coulomb. On complète les résultats semi-analytiques antérieurs obtenus dans Moirot, Nguyen, C. R. Acad. Sci. Paris Sér. IIb 328 (2000) 663-669, sur les ondes adhérence-glissement en simulant par le calcul numérique la transition dynamique du système à partir d'un état initial quelconque vers la propagation d'une onde de surface. Le cas des ondes adhérence-glissement-décollement est examiné.
\end{abstract}

\begin{abstract}
A numerical study on the dynamic response of a mechanical system composed of two coaxial cylinders in relative rotation and unilateral contact with Coulomb friction is presented. This discussion complements the semi-analytical results on stickslip waves given in Moirot, Nguyen, C. R. Acad. Sci. Paris Sér. IIb 328 (2000) 663-669, by some numerical simulations on the dynamic transition of the system from a given initial state to a surface wave. The case of stick-slip-separation waves is considered.
\end{abstract}

Mots-clés : Frottement ; Mécanique des solides numérique ; Contact unilatéral ; Onde adhérence-glissement-décollement

Keywords: Friction; Computational solid mechanics; Unilateral contact; Stick-slip-separation waves

Adresses e-mail : oueslati@lms.polytechnique.fr (A. Oueslati), son@lms.polytechnique.fr (Q.-S. Nguyen), Laurent.Baillet@insa-lyon.fr (L. Baillet). 


\section{Abridged English version}

The mechanical response in plane strain of an elastic cylinder, of internal radius $R$ and external radius $R^{*}$, in frictional contact on its inner surface with a rotating rigid cylinder of radius $R+d$ and of angular rotation $\Omega$ is considered when the displacement is maintained fixed on its outer surface and Coulomb's law of dry friction assumed with a constant friction coefficient $f$ on the inner surface. The mismatch $d$ is a load parameter controlling normal contact pressures. This problem has been discussed analytically in [1,2] with an interesting reduction of the governing equations. The existence of periodic responses under the form of stick-slip waves has been discussed within this approximation. Since the amplitude of these waves increases with the rotation, stick-slip solutions are no longer available when the rotation is strong enough and it has been expected that stick-slip-separation waves should occur. In order to have a better understanding on the dynamic response of the system in this case, a numerical study of the problem by the finite element method and by a direct integration has been performed and enables us to give the dynamic transition of the system from a given initial state to a cyclic limit response which may be a stick-slip wave or a stick-slip-separation wave. The obtained results concerning stick-slip-separation waves are presented in this Note.

An explicit scheme using Lagrange multipliers, as introduced in [8,9], for the frictional contact between a rigid obstacle and a deformable solid, is applied. For each time increment, the new coordinates of boundary nodes are first estimated as free nodes in a prediction step. If the condition of non-penetration is not satisfied, this step is followed by a correction step in which new appropriate reactions ensuring the contact are computed. A classical time-discretization is adopted following $\beta$-method with $0.5 \leqslant \beta_{2} \leqslant 1$, cf. [9]. The numerical results obtained with $\beta_{2}=0.9$ are presented here.

The case $(E / \rho)^{1 / 2}=10^{3} \mathrm{~m} / \mathrm{s}, v=0.3, R=0.25 \mathrm{~m}$ was considered. Starting from a motionless initial state, the normal displacement is then applied, increasing linearly from 0 to its final value. A limit cyclic response is then obtained for large time and consists of a stick-slip-separation wave in mode 4 for $f=0.7, d / R=0.001$ and $\Omega=50 \mathrm{rad} / \mathrm{s}$. The result is shown in Fig. 1 for radial displacements. The motion of a node is described in Fig. 2 . The propagation at constant celerity is observed in Fig. 3 . The computed celerity $730 \mathrm{~m} / \mathrm{s}$ is an intermediate value between the celerities of shear wave $c_{s}=620 \mathrm{~m} / \mathrm{s}$ and pressure wave $c_{p}=1160 \mathrm{~m} / \mathrm{s}$ of the material. The direction of propagation depends on the thickness ratio $R^{*} / R$. For example, these waves propagate in the rotation sense if $R^{*} / R \leqslant 1.15$ and in the opposite sense if $R^{*} / R \geqslant 1.25$, in accord with the semi-analytical prediction [2]. When $R^{*} / R \rightarrow 1$, the case of sliding contact of layers are recovered in the limit and the derived conclusion is in accord with recent results of the literature, $\mathrm{cf}$. [8,5,10], concerning the direction of wave propagation.

It is checked that a stick-slip wave is obtained when the rotation is slower. For example, if $f=0.3, d / R=$ 0.001 , a stick-slip wave results when $\Omega=1 \mathrm{rad} / \mathrm{s}$ instead of a stick-slip-separation wave when $\Omega=20 \mathrm{rad} / \mathrm{s}$, cf. Fig. 4. In this case, the wave profile of the semi-analytical approach [2] is approximately recovered.

The mode number increases when $R^{*} / R$ decreases. For example, when $\Omega=50 \mathrm{rad} / \mathrm{s}, f=0.7$ and $d / R=$ 0.001 , modes $2,8,12$ are obtained respectively for $R^{*} / R=3.5,1.5$ and 1.25 as shown in Fig. 5.

Some simulations with different initial data have been performed in order to study the sensibility of the results. It has been found that the limit regime may depend on the initial conditions.

This discussion contributes to new developments of the literature, cf. $[8,4,1,2,10]$ on the propagation of stickslip or stick-slip-separation waves in contact surfaces as an alternative to the unstable steady sliding solution.

\section{Problème de cylindres coaxiaux}

On s'intéresse dans cette communication à la réponse dynamique d'un solide élastique en contact unilatéral frottant avec un obstacle mobile. L'étude a pour objectif d'analyser par des simulations numériques la répartition et la propagation des zones de contact adhérent ou de glissement ou de séparation entre deux cylindres coaxiaux. Ce modèle de cylindres coaxiaux a été introduit et discuté analytiquement, cf. [1] ou [2], en vue de la modélisation du 
phénomène de crissement des freins. On rappelle que la réponse dynamique et la propagation des ondes de surface jouent un rôle important dans l'analyse de nombreux problèmes de la Mécanique du Contact (moteur à ultrason [3], prévision et simulation sismique, vibration et instabilité engendrées par le frottement [4-7]).

Il s'agit de la réponse dynamique en déformation plane d'une enveloppe cylindrique élastique $C$, de rayons interne $R$ et externe $R^{*}$, en contact avec frottement sur la surface interne $S$ avec un cylindre rigide de rayon $R+d$, en mouvement de rotation à la vitesse angulaire $\Omega$, la surface externe $S^{*}$ étant maintenue fixe. La loi de Coulomb, à coefficient de frottement constant $f$, est admise sur la surface interne. Les équations décrivant la réponse dynamique de l'enveloppe correspondent aux relations classiques de l'élasticité avec les conditions aux limites données et les conditions de contact unilatéral avec frottement :

$$
\left\{\begin{array}{l}
\forall x \in C: \quad \epsilon=(\nabla \mathbf{u})_{s}, \quad \operatorname{Div} \sigma=\gamma \ddot{\mathbf{u}}, \quad \sigma=\lambda \operatorname{tr}(\epsilon) I+2 \mu \epsilon \\
\forall x \in S^{*}: \quad u=0, \quad v=0 \\
\forall x \in S: \quad u-d \geqslant 0, \quad p \geqslant 0, \quad p(u-d)=0, \quad|q| \leqslant f p, \quad q(R \Omega-\dot{v})-f p|R \Omega-\dot{v}|=0
\end{array}\right.
$$

où $p$ et $q$ désignent respectivement les réactions normale et tangentielle. Une approche semi-analytique a été développée, cf. [2], en résolvant un système d'équations réduit obtenu par une condensation statique. Cette approche a permis de mettre en évidence l'existence des ondes adhérence-glissement et de donner des estimations intéressantes concernant leurs caractéristiques comme les célérités, les fréquences, les amplitudes, les profils des vitesses et des réactions. En particulier, l'amplitude des ondes croît avec la vitesse de rotation du cylindre rigide de sorte que la possibilité de décollement n'est pas exclue lorsque la réponse en onde adhérence-glissement n'est plus valable. Comme le calcul semi-analytique n'est pas envisageable dans un tel cas compte tenu de la complexité des équations réduites, nous présentons dans cette note une approche purement numérique par la méthode des éléments finis et par intégration directe des équations. Cette approche numérique devrait compléter les résultats antérieurs dans plusieurs directions, en particulier pour l'obtention des ondes adhérence-glissement-décollement.

\section{Approche numérique}

La méthode numérique mise en oeuvre est une méthode dynamique explicite, compatible avec l'introduction des liaisons traduisant le contact frottant [8,9]. Plusieurs algorithmes de gestion du contact avec multiplicateurs de Lagrange ont été développés dans le code de calcul PLAST2 par Baillet [8], en particulier pour traiter le contact entre une surface rigide et un corps déformable. Les équations du système sont écrites sous la forme

$$
\left\{\begin{array}{l}
M \ddot{u}_{m}+K u_{m}+G_{m+1}^{\mathrm{T}} \lambda_{m}=F_{m} \\
G_{m+1}\left(u_{m+1}+X\right)=0
\end{array}\right.
$$

dans laquelle $F_{m}$ désigne les efforts donnés, $G_{m+1}$ les liaisons de contact et $\lambda_{m}$ les multiplicateurs de Lagrange associés, représentant les réactions normales et tangentielles. Les vitesses et accélérations actuelles $\dot{u}_{m}$ et $\ddot{u}_{m}$ s'expriment à chaque pas de temps h en fonction des déplacements à l'instant suivant par un schéma de discrétisation (dit méthode $\beta$, bien connu en calcul des structures)

$$
\left\{\begin{array}{l}
\dot{u}_{m}=\frac{1}{1+2 \beta_{2}}\left\{\dot{u}_{m-1}+h\left(1-\beta_{2}\right) \ddot{u}_{m-1}+\frac{2 \beta_{2}}{h}\left(u_{m+1}-u_{m}\right)\right\} \\
\ddot{u}_{m}=\frac{2}{h^{2}}\left(u_{m+1}-u_{m}-h \dot{u}_{m}\right)
\end{array}\right.
$$

dépendant du paramètre $0.5 \leqslant \beta_{2} \leqslant 1$, le schéma des différences centrées classique correspond d'ailleurs à $\beta_{2}=0.5$. Pour chaque incrément de temps, les nouvelles coordonnées $X+u_{m+1}^{*}$ des noeuds frontières du corps déformable sont calculées en supposant d'abord que les efforts de contact sont nuls. Par exemple, pour $\beta_{2}=0.5$, on a

$$
u_{m+1}^{*}=h^{2} M^{-1}\left(-K u_{m}+F_{m}\right)+2 u_{m}-u_{m-1}
$$


Cette étape de prédiction est ensuite corrigée, pour les noeuds violant la condition de non-interpénétration, par un calcul des forces de contact nécessaires pour les ramener sur la surface rigide et par des itérations internes pour écrire les liaisons de contact $G_{m+1}$,

$$
\left\{\begin{array}{l}
\lambda_{m}=\left[h^{2} G_{m+1} M^{-1} G_{m+1}^{\mathrm{T}}\right]^{-1} G_{m+1}\left(u_{m+1}^{*}+X\right) \\
u_{m+1}^{c}=-h^{2} M^{-1} G_{m+1}^{T} \lambda_{m} \\
u_{m+1}=u_{m+1}^{*}+u_{m+1}^{c}
\end{array}\right.
$$

On répète cette étape pour satisfaire la loi de Coulomb. Si $u_{m n}, \lambda_{m n}$ et $\lambda_{m t}$ désignent respectivement la distance normale et les vitesses relatives (normale et tangentielle) d'un noeud du solide déformable par rapport au solide rigide, un noeud en contact $\left(u_{m n}=0\right)$ est adhérent si $\lambda_{m n}>0$ et si $\left|\lambda_{m t}\right|<f \lambda_{m n}$, glissant si $\lambda_{m n} \geqslant 0$ et si $\left|\lambda_{m t}\right|=f \lambda_{m n}$. Pour un noeud glissant, il faut que $\lambda_{m t}$ et $\dot{u}_{n t}$ soient de signe opposé.

Le calcul a été effectué avec $\beta_{2}=0,9$ qui assure un grand amortissement numérique. Cet amortissement n'est pas gênant pour le calcul du cycle limite car l'énergie est continuellement injectée dans le système par la rotation imposée au cylindre rigide. Il a pour effet d'accélérer artificiellement la convergence vers cet état. On a vérifié que la même convergence est pratiquement obtenue pour des valeurs de $\beta_{2}$ supérieures à 0,60 . La vitesse de convergence est par contre nettement plus lente lorsque $\beta_{2}$ est proche de 0,50 . La convergence par rapport au maillage a été testée avec des maillages 5 fois plus denses. Dans l'intervalle $0 \leqslant f \leqslant 1$, le coefficient de frottement a peu d'influence sur la vitesse de convergence.

\section{Ondes adhérence-glissement-décollement}

On présente dans cette section les résultats numériques obtenus sur les ondes adhérence-glissementdécollement pour un cylindre de caractéristiques élastiques $E=2500 \mathrm{Mpa}, v=0,3, \rho=2500 \mathrm{~kg} / \mathrm{m}^{3}$ et de rayon intérieur $R=0,25 \mathrm{~m}$.

Partant d'un état initial de position et de vitesse donné, le chargement (en déplacement ou en rotation) est ensuite appliqué progressivement pour atteindre à partir d'un certain instant les valeurs imposées $d$ et $\Omega$. La réponse du système tend très vite vers un cycle limite représentant une onde adhérence-glissement ou adhérence-glissementdécollement périodique comportant plusieurs périodes spatiales sur le cercle de contact. Il s'agit d'une onde en mode $k$ si la réponse limite comporte $k$ périodes.

On vérifie d'abord qu'une onde adhérence-glissement-décollement est effectivement obtenue lorsque la vitesse de rotation est suffisamment importante. Par exemple, pour $\Omega=50 \mathrm{rad} / \mathrm{s}, f=0,7$ et $d / R=0,001$, une onde adhérence-glissement-décollement en mode 4 est obtenue comme l'indique la carte du déplacement radial de la Fig. 1. Le mouvement d'un point matériel est donné sur la Fig. 2. La propagation à vitesse constante $730 \mathrm{~m} / \mathrm{s}$ et la convergence vers une réponse périodique sont illustrées sur la Fig. 3 par les variations au cours du temps de la pression normale et du déplacement radial.

Pour $d / R=0,005, f=0,3$ et $\Omega=10 \mathrm{rad} / \mathrm{s}$, une onde adhérence-glissement en mode 4 est obtenue. Dans ce cas, les résultats numériques sont très proches des résultats semi-analytiques [2].

\subsection{Sens et vitesse de propagation}

On constate que les ondes se propagent dans le sens de la rotation lorsque $R^{*} / R \leqslant 1,15$ et dans le sens opposé pour $R^{*} / R \geqslant 1,25$, conformément aux analyses semi-analytiques. D'après ces analyses [2], le sens de propagation est régi par le signe d'un paramètre de couplage entre les déplacements radiaux et tangentiels de la surface de contact. Pour $R^{*} / R \rightarrow 1$, on obtient à la limite le contact d'une couche élastique plane avec un demi-plan rigide. Il est intéressant de comparer dans ce cas notre conclusion concernant le sens de propagation des ondes aux résultats de la littérature [8,5,10]. Dans [5], une perturbation dynamique de la solution de glissement stationnaire va devenir 

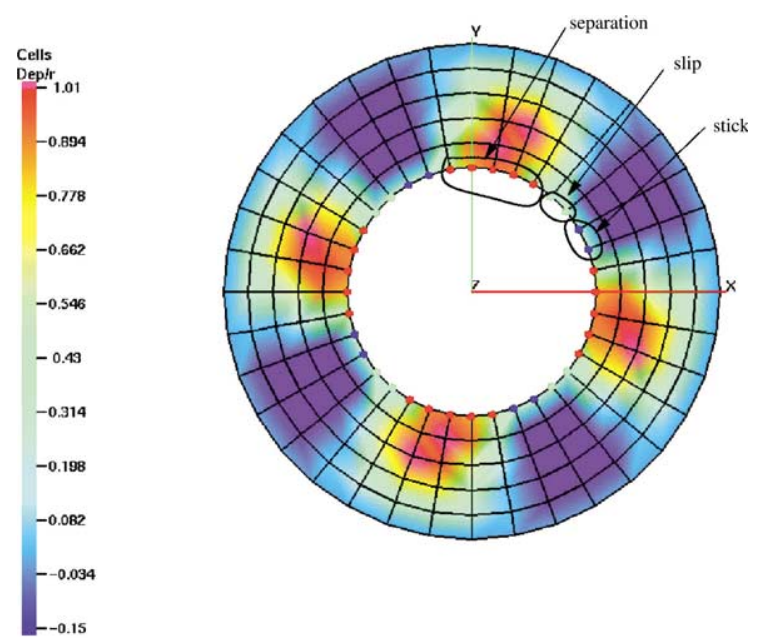

Fig. 1. Résultats numériques illustrant une onde adhérence-glissement-décollement en mode 4. Cette figure présente les isovaleurs du déplacement radial en mm. Les zones de décollement, de glissement et d'adhérence y sont indiquées respectivement en rouge, vert et bleu.

Fig. 1. Numerical results illustrating a stick-slip-separation wave in mode 4. The isovalues of radial displacements are given in mm. Separation, slip and stick zones of the inner surface are respectively colored in red, green and blue.
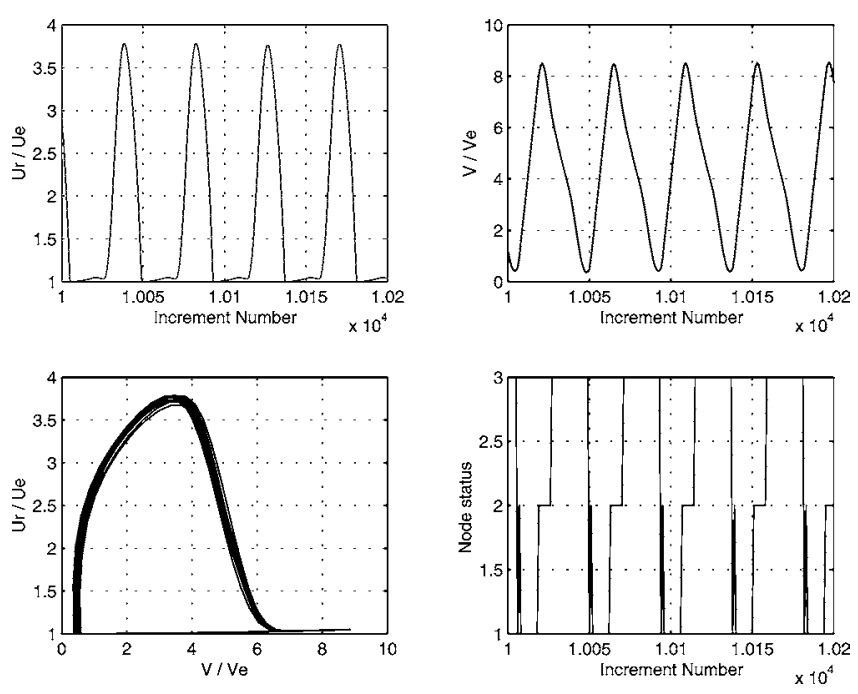

Fig. 2. Résultats numériques illustrant le mouvement d'un point matériel appartenant à la surface $S$. Cette figure donne la trajectoire, les déplacements radial $U$ et tangentiel $V$ ainsi que la nature du contact $(1=$ adhérence, $2=$ glissement, $3=$ décollement) au cours du temps (un incrément $=1,35 \times 10^{-5}$ seconde). L'indice e se réfère à la solution de glissement stationnaire.

Fig. 2. Numerical results illustrating the motion of a material point of the inner surface. The trajectory and the variations of radial and tangential displacements and of the contact nature $\left(1=\right.$ stick, $2=$ slip, $3=$ separation) are given with respect to time (a time increment $=1.35 \times 10^{-5}$ second). The subscript e refers to the steady sliding solution.

une onde à croissance exponentielle se propageant dans le sens du mouvement du demi-plan rigide. Pour le cas d'un solide rectangulaire bi-dimensionnel [10] ou tri-dimensionnel [8] entrainé par un demi-plan rigide, il a été observé que les ondes adhérence-glissement-décollement prennent naissance sur le bord d'attaque et se propagent suivant le mouvement du demi-plan. Notre conclusion est donc en parfait accord avec ces résultats. 


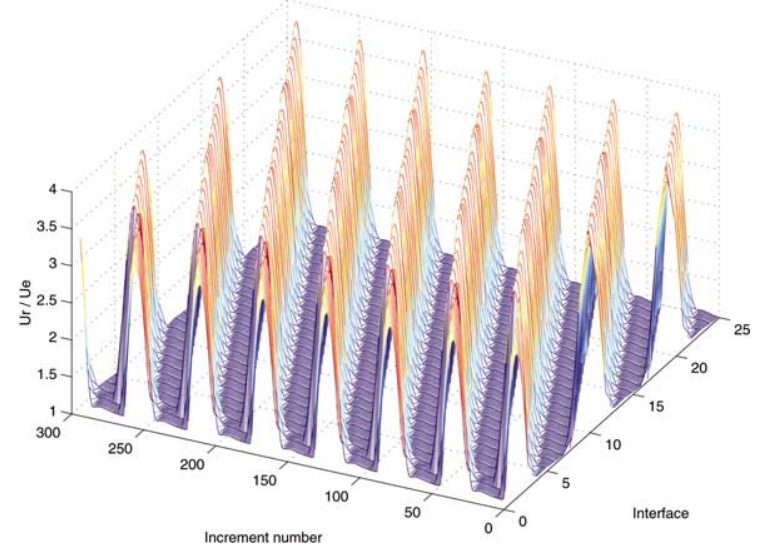

(a)

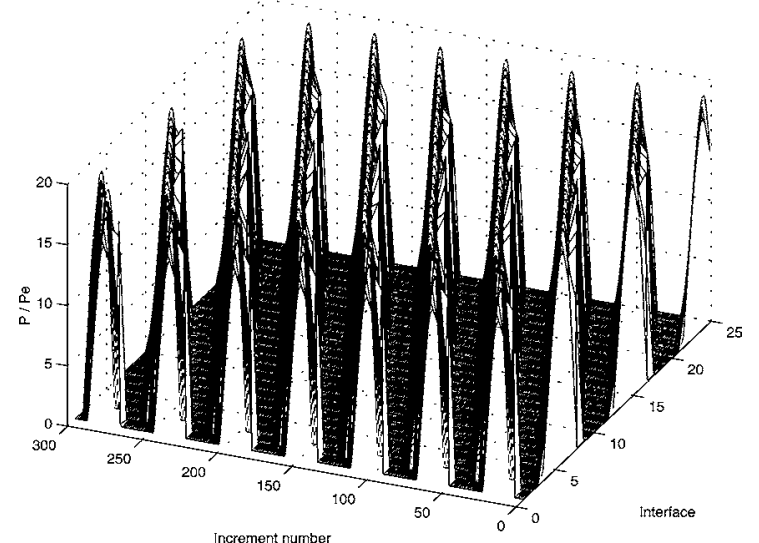

(b)

Fig. 3. Transition vers une onde adhérence-glissement-décollement en mode 4. Courbes donnant les variations du déplacement radial et de la pression normale au cours du temps.

Fig. 3. Transition to a stick-slip-separation wave in mode 4. Time variations of the radial displacements and normal pressures on the inner surface.
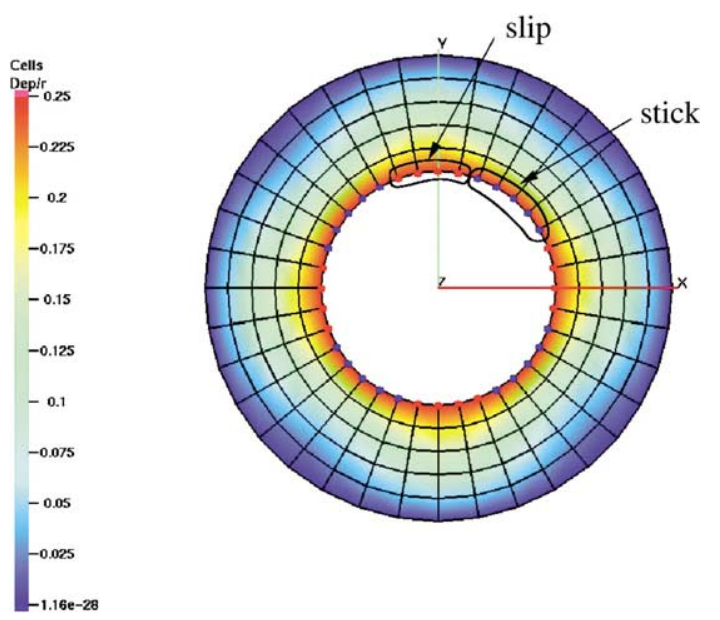

(a)

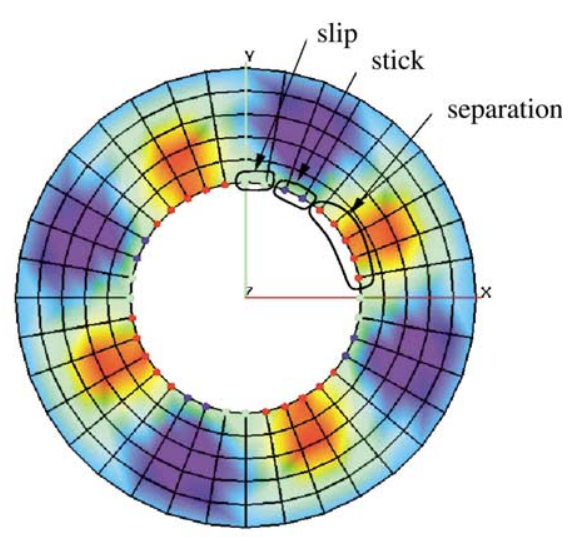

(b)

Fig. 4. Influence de la vitesse de rotation : cartes de déplacement radial (en $\mathrm{mm}$ ) pour $\Omega=1$ et $20 \mathrm{rad} / \mathrm{s} \mathrm{respectivement.}$

Fig. 4. Influence of the rotation: radial-displacement maps (in $\mathrm{mm}$ ) for $\Omega=1$ and $20 \mathrm{rad} / \mathrm{s}$ respectively.

Les vitesses de propagation associées sont voisines de la célérité des ondes transversales $c_{s}=620 \mathrm{~m} / \mathrm{s}$ et restent inférieures à la célérité des ondes longitudinales $c_{p}=1160 \mathrm{~m} / \mathrm{s}$ dans le solide.

\subsection{Influence de la rotation}

L'amplitude des ondes crôt avec la vitesse de rotation imposée. Ainsi, pour $d / R=0,001$, une onde adhérenceglissement en mode 4 résulte lorsque $\Omega=1 \mathrm{rad} / \mathrm{s}$. Elle devient une onde adhérence-glissement-décollement en mode 4 lorsque $\Omega=20 \mathrm{rad} / \mathrm{s}$, cf. Fig. 4 . Pour des vitesses de rotation évanessantes, ce sont des oscillations de faibles amplitudes autour de la solution de glissement stationnaire. 


\subsection{Influence de la pression de contact}

Le déplacement radial imposé $d$ permet de contrôler la pression de contact. Le décollement, empêché par une forte pression de contact, disparaît lorsque la pression de contact est suffisamment forte. Ainsi, avec $f=0,4$, $\Omega=10 \mathrm{rad} / \mathrm{s}$, une onde adhérence-glissement-décollement en mode 4 est obtenue pour $d / R=0,005$ à la place d'une onde adhérence-glissement lorsque $d / R=0,0005$.

\subsection{Influence de l'épaisseur}

Le mode observé croît lorsque l'épaisseur du cylindre décroît. Par exemple, lorsque $\Omega=50 \mathrm{rad} / \mathrm{s}, d / R=$ 0,001, $f=0,7$, les modes 2, 8 et 12 sont respectivement obtenus pour $R^{*} / R=3,5,1,5$ et 1,25 comme l'indique la Fig. 5 avec des vitesses de propagation associées $470,700,830 \mathrm{~m} / \mathrm{s}$. Pour $R^{*} / R=1,15, D$ étant positif, la propagation change de sens.
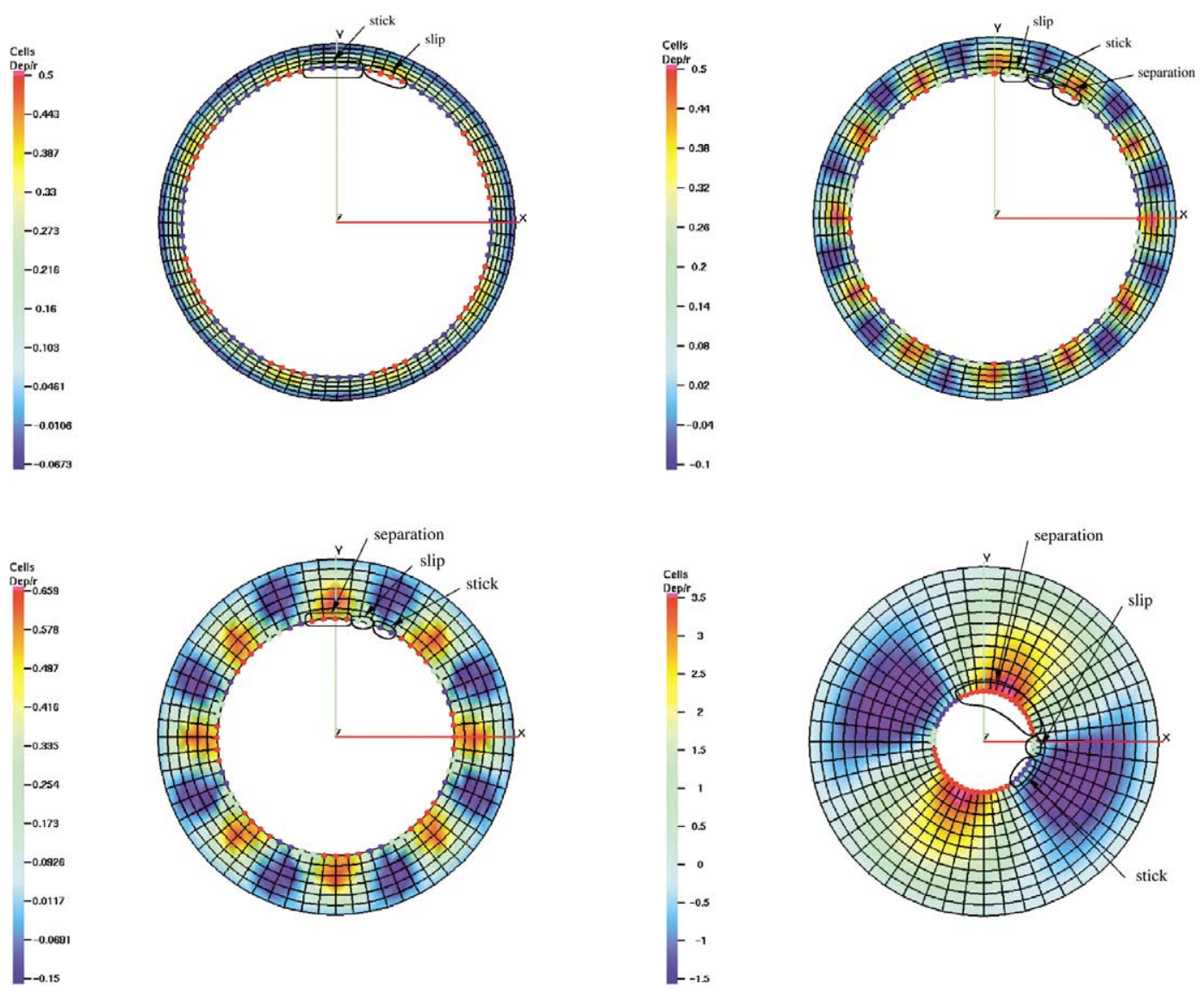

Fig. 5. Influence de l'épaisseur. Tracé des isovaleurs du déplacement radial en mm.

Fig. 5. Influence of the thickness: Isovalues (in $\mathrm{mm}$ ) of the radial displacements. 


\subsection{Transition vers le cycle limite}

La transition vers un cycle limite s'effectue en un temps très court, inférieur à $0,1 \mathrm{sec}$. Le cycle limite pourrait a priori dépendre de l'état initial. Cette dépendance semble néammoins faible car les modes calculés sont relativement stables par rapport aux données initiales.

\section{Conclusion}

Dans ce papier, nous avons présenté un exemple d'onde adhérence-glissement-décollement. Cette réponse cyclique est obtenue numériquement par une méthode d'intégration explicite. Elle contribue aux résultats de la littérature $[8,4,1,2,10,3]$ sur les caractéristiques des ondes de contact, en particulier sur les réponses dynamiques cycliques conséquentes à l'instabilité du glissement stationnaire.

\section{Références}

[1] F. Moirot, Étude de la stabilité d'un équilibre en présence du frottement de Coulomb. Application au crissement des freins à disque, Thèse, École Polytechnique, Paris, 1998.

[2] F. Moirot, Q.S. Nguyen, An example of stick-slip waves, C. R. Acad. Sci. Paris Sér. IIb 328 (2000) 663-669.

[3] O.Y. Zharii, Frictional contact between the surface wave and a rigid strip, J. Appl. Mech. 63 (1996) 15-20.

[4] J.A.C. Martins, S. Barbarin, M. Raous, A. Pinto da Costa, Dynamic stability of finite dimensional linear elastic system with unilateral contact and Coulomb's friction, Comput. Methods Appl. Mech. Engrg. 177 (1999) 298-328.

[5] J.A.C. Martins, J. Guimaraes, L.O. Faria, Dynamic surface solutions in linear elasticity and viscoelasticity with frictional boundary conditions, J. Vib. Acoustics 117 (1995) 445-451.

[6] Q.S. Nguyen, Stability and Nonlinear Solid Mechanics, Wiley, Chichester, 2000.

[7] J.T. Oden, J.A.C. Martins, Models and computational methods for dynamic friction phenomena, Comput. Methods Appl. Mech. Engrg. 52 (1985) 527-634.

[8] L. Baillet, Y. Berthier, O. Bontemps, M. Brunet, Tribologie de l'interface fibre/matrice. Approche théorique et expérimentale, Rev. Composites et Matériaux Avancés 7 (1997) 89-105.

[9] N.J. Carpenter, R.L. Taylor, M.G. Katona, Lagrange constraints for transient finite element surface contact, Int. J. Numer. Methods Engrg. 32 (1991) 103-128.

[10] Y. Renard, Modélisation des instabilités liées au frottement sec des solides, aspects théoriques et numériques, Thèse de doctorat, Université de Grenoble, 1998. 